American Medical Journal 3 (2): 141-146, 2012

ISSN 1949-0070

C 2012 Science Publications

\title{
State of the Art: Practical Approach for Diagosis of Pulmonary Embolism
}

\author{
Narat Srivali, Supawat Ratanapo, \\ Promporn Suksaranjit, Wisit Cheungpasitporn and Daych Chongnarungsin \\ Department of Internal Medicine, Bassett Medical Center and Columbia University, \\ College of Physicians and Surgeons, Cooperstown, New York
}

\begin{abstract}
Pulmonary embolism is one of the leading causes of cardiovascular related death beside myocardial infarction and stroke. Contrary to stroke and myocardial infarction, diagnosis of pulmonary embolism is considered to be much more difficult and complicated compare with the treatment once diagnosis is confirmed. Multiple diagnostic studies including clinical scoring, serum biomarker and diagnostic imaging were developed to help clinician rule in or rule out pulmonary embolism. In this article, we discussed the advantage and disadvantage of these tests and a practical way to choose the right test to diagnose pulmonary embolism.
\end{abstract}

Key words: Pulmonary embolism, D-dimer, myocardial infarctio, pulmonary embolism

\section{INTRODUCTION}

The term pulmonary embolism refers to blockage in the pulmonary artery or its branches which has various etiologies. This review focuses on the diagnosis of thromboembolic pulmonary embolism which is the most common cause of pulmonary embolism encounter in clinical practice.

Pathophysiology: Acute pulmonary embolism is usually caused by dislodgement of the thrombus that forms in deep venous system of lower extremities. The thrombus flows through venous system, entering right ventricle and occlude pulmonary artery or its branches. The burden from the pulmonary thrombus correlates well with severity of clinical manifestation and urgency in patient management. In general, obstruction of pulmonary artery produces 3 effects.

Right ventricular dysfunction: Occlusion of pulmonary artery increases afterload of right ventricle leading to right ventricular dysfunction. Depends on severity of occlusion, the degree of right ventricular dysfunction is varied. Complete occlusion will cause sudden hemodynamic collapse or cardiac arrest since zero cardiac output from right side would also lead to zero cardiac output from the left. The lesser degree of occlusion would lead to acute right ventricular strain. This would increase right ventricular tension and relative increase in myocardial oxygen demand which will eventually lead to right ventricular myocardial ischemia. This explains why the patient may complain of angina pain or has elevated cardiac biomarkers.

Perfusion and ventilation mismatch: The decrease in lung perfusion may cause pulmonary infarction. This condition is usually rare $(<10 \%)$ because lung parenchyma has 3 sources of oxygen supply: pulmonary artery, brachial artery and direct oxygen delivery from the airways (Bjork and McNeil, 1977). Impairment of pulmonary artery perfusion creates alveolar dead which would eventually lead to arterial hypoxemia.

Resolution of embolism: Various natural mechanisms will lead to clot resolution. These mechanisms are fibrinolysis and organization with recanalization or both. The imbalance between these two processes may lead to highly morbid condition known as chronic thromboembolic pulmonary hypertension.

Diagnosis: Unfortunately, the use of history and physical findings were shown to be unreliable and nonspecific to diagnose pulmonary embolism. In order to make a diagnosis, clinicians need to have high index of suspicion and have clear knowledge of sensitivity and specificity of various diagnostic tools.

Clinical manifestation: The symptoms of pulmonary embolism differ for each patient (Fig. 1 and 2). Dyspnea at rest or exertion is the most common complaint.

Corresponding Author: Narat Srivali, Department of Internal Medicine, Bassett Medical Center and Columbia University, College of Physicians and Surgeons, Cooperstown, New York 


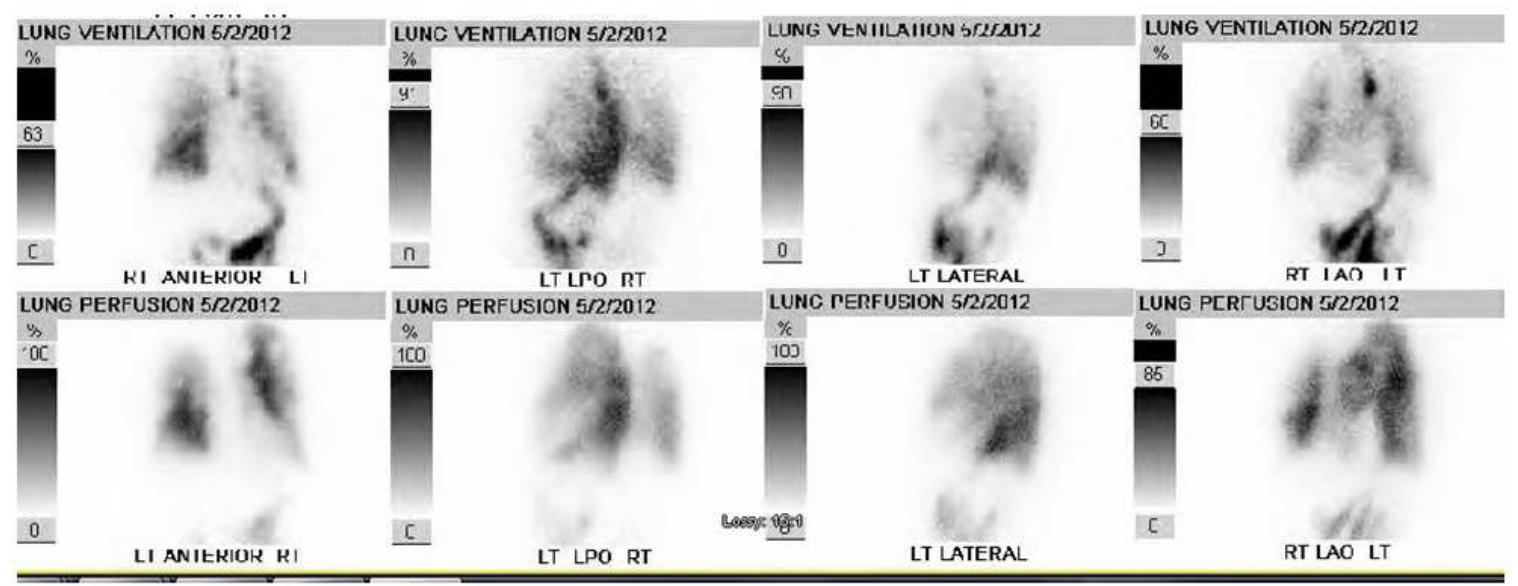

Fig. 1: V/Q scan from 64 year old female who present with chest pain. Due to her chronic kidney disease, V/Q scan was chosen as diagnostic strategy for pulmonary embolism. The lower row demonstrates perfusion scintigraphywith multiple defects which are not presented in ventilation scintigraphy in the upper row (mismatched defect.) It is reported as high probability for pulmonary embolism

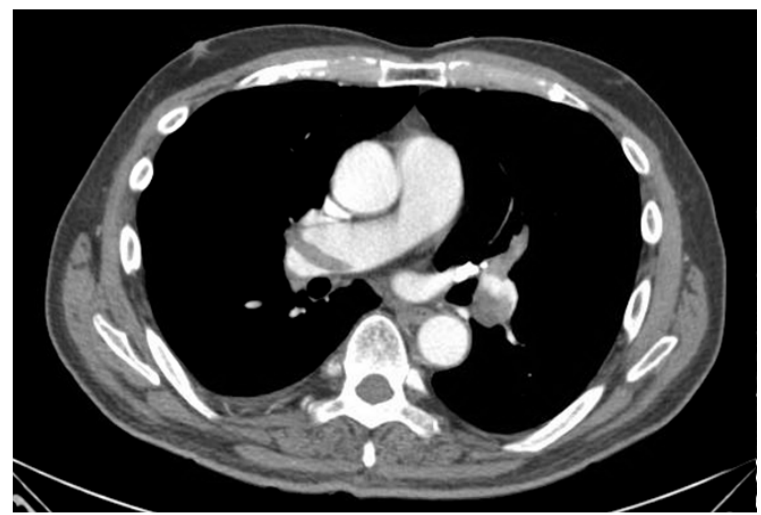

(a)

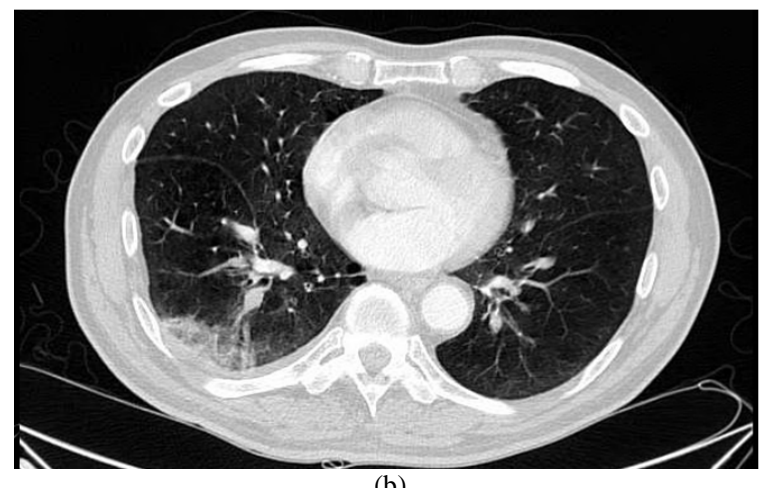

(b)

Fig.2: CT pulmonary angiography from 65-year-old male presented with shortness of breath and right side pleuritic chest pain (a) Filling defect is seen in right pulmonary artery (b) Lung window shows area of pulmonary infarction in right lower lobe
Table 1: Clinical manifestation of pulmonary embolism in patient without prior cardiopulmonary disease from PIOPED study

\begin{tabular}{ll}
\hline Symptom & Frequency $(\%)$ \\
\hline Dyspnea & 73 \\
Orthopnea (>2 pillows) & 37 \\
Pleuritic pain & 58 \\
Chest pain & 25 \\
Cough & 45 \\
Wheezing & 27 \\
Calf or thigh swelling & 52 \\
\hline
\end{tabular}

Pleuritic chest pain is usually due to pulmonary infarction leading to irritation of parietal pleura. It usually seen in patient with small embolism located in small branch of pulmonary artery. Patient who has significant rise in right ventricular pressure, which leads to increase myocardial wall tension and myocardial ischemia, will have angina like chest pain. Syncope and hypotension are due to drop in cardiac output from large thromboembolism. Hemoptysis may be a presenting symptom but usually modest. The frequency of each symptom is shown in Table 1 (Stein et al., 1991; Ryu et al., 1998).

Various objective findings are described in patients with pulmonary embolism. They are also not helpful because they can be found in other diseases. The most common signs were unexplained tachypnea (70\%), tachycardia (30\%), decreased breath sounds, rales (30\%), increase accentuation of second heart sound (loud P2) and jugular venous distention which can be seen in various disease states. Hypotension, present of right-sided S3 gallop and a parasternal lift usually indicate right ventricular failure from massive pulmonary embolism. Fever may also develop but rarely exceeds 38.3 degree Celsius. 
Due to non-specificity of clinical manifestation, differential diagnosis of pulmonary embolism is broad which include congestive heart failure, exacerbation of chronic lung disease, postoperative atelectasis, viral pleurisy and pneumonia. Clinician should be well aware of the fact that clinical presentation of pulmonary embolism may often be subtle and nonspecific. Reliance on subjective and objective data may lead to delay diagnosis and unnecessary morbidity and mortality.

Clinical probability assessment: The first step in diagnosis of pulmonary embolism is always assessing the pretest probability. The pretest probability has great effect on the positive predictive value and negative predictive value of imaging study which are considered to be the best diagnostic tool to date. In patient with low clinical probability and negative D-dimer, clinician can be confidently rule out pulmonary embolism without further imaging studies. The most commonly use scoring system for the patient admitted to the hospital is the modified Wells criteria. It divides patients in to 2 group (PE likely and PE unlikely) bases on simple clinical indicator and no laboratory studies are needed in the calculation. Patient with score of more than 4 are classified as PE likely.

Diagnostic tests: Various diagnostic studies were used to aid in diagnosis of pulmonary embolism. Clinician need to have knowledge of quality of each test to avoid over and under diagnosis. Judicious use of diagnostic algorithm is proved to be the most effective strategy in reaching the diagnosis and to avoid unnecessary diagnostic study in the low risk population.

Electrocardiography: Historically, various electrocardiographic finding were claimed to be highly suggestive of pulmonary embolism which include S1Q3T3 pattern (deep S wave in lead I, Q wave and inverted $\mathrm{T}$ wave in lead III), right ventricular strain and new incomplete right bundle branch block. These findings were later found to be infrequent during acute pulmonary embolism but more common among patients with massive pulmonary embolism (Chan et al., 2001). Some of the ECG findings are also proved to be a useful prognostic indicator. In the analysis of 386 patients with proved pulmonary embolism, the presence of right ventricular strain (complete or incomplete right ventricular branch block, S1Q3T3 and negative T wave in V1-V4) was associated with adverse outcome independently of echocardiographic finding (Vanni et al., 2009).

D-Dimer: D-dimer is a degradation product of crosslinked fibrin. Its concentration increases when thrombosis occurs. It has a high negative predictive value. Combining with clinical probability scoring system (Well score), patient with low pretest likelihood and negative D-dimer can be certainly ruled out and obviate the need for further diagnostic testing. There are various assays for D-dimer measurement and it is important for clinicians to know what assay is currently used in their institution because different assay has different negative predictive value. The use of quantitative ELISA assay provides a sensitivity of more than 95\% (Righini et al., 2008). This falls to approximately $90 \%$ with quantitative latex agglutination assay and $86 \%$ with semi-quantitative latex agglutination assay (Stein et al., 2004). However, multiple conditions may lead to elevation of D-dimer level which makes the test non-specific and provide little help in diagnosis when showing positive result. These conditions are arterial thromboembolic disease (such as myocardial infarction, atrial fibrillation or intracardiac thrombus), severe infection, recent surgery, severe liver disease, malignancy, D-dimer is also helpful to predict the risk of recurrence in patient who completed the treatment of pulmonary embolism with anticoagulant therapy.

Chest radiography: Various studies have shown that chest radiography is unreliable in the diagnosis of pulmonary embolism (Greenspan et al., 1982). PIOPED study found that abnormalities seen on the chest radiographs of patients with proven $\mathrm{PE}$ were seen with similar frequency in patients with no proven pulmonary embolism (Worsley et al., 1993). Chest radiography should be considered as a test to exclude other conditions rather than helping in diagnosis of pulmonary embolism. Various findings have been described in patient with pulmonary embolism:

- Peripheral oligemia (Westermark's sign) (Krishnan and Barrett, 2012) which shows absence of vascular marking in one area of lung parenchyma

- Peripheral airspace opacification representing pulmonary hemorrhage

- Linear atelectasis: This finding caused by ischemic injury that affects surfactant production

- Hampton's hump: A wedge-shaped opacity caused by pulmonary infarction

- Consolidation

- Pleural effusion: This may be seen in up to $50 \%$ of patients

Venous duplex scan: Treatment for lower extremities deep vein thrombosis and pulmonary embolism without hemodynamic compromise are basically the same. 
Following this principle, venous duplex scan is performed some patients with suspected pulmonary embolism since this test is considered to be non invasive and no need for contrast administration. However, before applying this strategy to patient care, clinician should be aware of the fact that only $29 \%$ of patients with pulmonary embolism had deep venous thrombosis detected by venous duplex scan (Turkstra et al., 1997).

Ventilation-perfusion scanning: Despite its decreasing popularity compare to $\mathrm{CT}$ scan, this test provide imaging study without the need of contrast administration which is ideal for patients with renal impairment or contrast allergy. This test has 2 parts: perfusion $(\mathrm{Q})$ scintigraphy to assess the distribution of pulmonary blood flow and Ventilation (V) scintigraphy. Ideally, patient who has pulmonary embolism without pulmonary infarction will have defect in the perfusion imaging and normal finding in ventilation imaging (V/Q mismatch.) However, if the area affected by pulmonary embolism becomes infarcted, a defect may be seen in both ventilation and perfusion study and the defect will become 'matched.'The result of V/Q scan is usually report as normal, low, intermediate and high probability for pulmonary embolism.

The accuracy of this test was extensively investigated in 2 large prospective studies which compare V/Q scan with pulmonary angiography: PIOPED (1990) and PISA-PED (Miniati et al., 1996). Both trials confirm that, a negative study rules out the diagnosis of pulmonary embolism with the same degree of certainty as a negative pulmonary angiogram. In PIOPED study, a high-probability study is associated with pulmonary embolism in $87 \%$ of patient. However, some findings in PIOPED study needs to be taken in to consideration when sending the patients for this test: (1) it was found that most of the patients didn't have a finding that fall in to category of negative or highprobability group which seem to be the categories that can be considered 'definitive' for ruling out or ruling in pulmonary embolism; (2) most of patients who had pulmonary embolism did not have a high-probability finding on V/Q scan; (3) majority of patients who didn't have pulmonary embolism didn't have a normal scan. Table 3 shows the accuracy of diagnosis of pulmonary embolism when V/Q scan is used in conjunction with clinical probability. From the table, if the patients fell in to the shaded area the exclusion or diagnosis of pulmonary embolism will be impossible thus limiting the utility of V/Q scan.

Spiral CT scan: Due to commonly non-diagnostic result from V/Q scan, chest CT scan has supplanted V/Q scan as initial imaging of choice in patient with suspected pulmonary embolism.
Table 2: Modified well score

\begin{tabular}{ll}
\hline Indicators & Score \\
\hline $\begin{array}{l}\text { Clinical symptoms of DVT } \\
\text { (leg swelling, pain with palpation) }\end{array}$ & 3.0 \\
$\begin{array}{l}\text { Other diagnosis less likely than } \\
\text { pulmonary embolism }\end{array}$ & 3.0 \\
Heart rate $>100$ & 1.5 \\
Immobilization ( $\geq 3$ days) or surgery & 1.5 \\
in the previous four weeks & \\
Previous DVT/PE & 1.5 \\
Hemoptysis & 1.0 \\
Malignancy & 1.0 \\
\hline
\end{tabular}

Table 3: Likelihood of pulmonary embolism according to finding from V/Q scan and clinical probability from PIOPED study. (Please note that the clinical probability used in this trial is not modified Well score)

\begin{tabular}{lllc}
\hline Finding from & \multicolumn{2}{c}{ Clinical probability $(\%)$} & Low \\
V/Q scan & High & Intermediate & 56 \\
High & 95 & 86 & 15 \\
Intermediate & 66 & 28 & 4 \\
Low & 40 & 15 & 2 \\
Normal & 0 & 6 &
\end{tabular}

Table 4: Positive predictive value and negative predictive value of spiral CT in the diagnosis of pulmonary embolism. Data from PIOPED II study

\begin{tabular}{llll}
\hline & \multicolumn{3}{c}{$\begin{array}{l}\text { Clinical } \\
\text { probability }\end{array}$} \\
Variable & High (\%) & Intermediate (\%) & Low (\%) \\
\hline Positive predictive value & 96 & 92 & 58 \\
Negative predictive value & 60 & 89 & 96 \\
\hline
\end{tabular}

It has many advantages over V/Q scan which are: (1) higher specificity; (2) more readily available; (3) allow diagnosis of other disease process; (4) faster to obtain and (5) easier to use in emergency situation (Schoepf $e t$ al., 2004). Few contraindications for spiral CT scan are renal insufficiency, anaphylaxis to intravenous contrast agent and pregnancy. The accuracy of spiral CT scan was demonstrated in PIOPED II study with the sensitivity of $83 \%$ and specificity of $90 \%$ (Stein et al., 2006). When combining with pretest clinical probability score (i.e. Well's score), the positive predictive value and negative predictive value are shown in Table 4.

Clinicians need to pay extra caution when applying the result of the CT scan that has discordant result with clinical probability i.e., negative study in patient with high clinical probability and positive study in patient with low clinical probability. As demonstrate in the shaded area of Table 4, these groups of patient has much lower negative predictive value and positive predictive value compare with other groups. Other practical points that need to be taken in to consideration when interpreting spiral CT are: (1) the type and the resolution of the scanner, (2) adequacy of the bolus of injected contrast material, (3) whether CT protocol specific for PE was used versus an aortic dissection protocol and (4) other diagnoses that may explain the symptoms. 


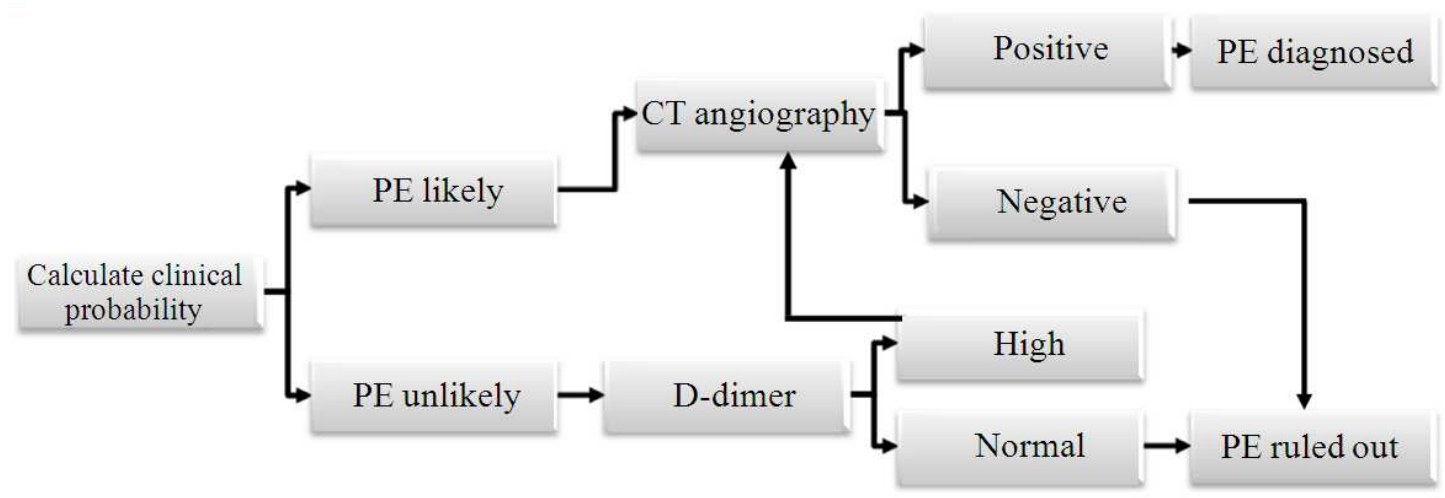

Fig. 3: Diagnostic algorithm of pulmonary embolism in Christopher study

Algorithms for pulmonary embolism diagnosis: As describe above, multiple diagnostic tools are available for diagnosis of pulmonary embolism each of them has advantage and disadvantage and it can be very confusing to clinicians when choosing the test that is best for their patient. To decrease this confusion, diagnostic algorithm has been developed and was tested in large prospective clinical study. Christopher study is a well-known study that tests the algorithm that combine modified Wells criteria, use of D-dimer and spiral CT scan to arrive at a diagnosis of pulmonary embolism (Belle et al., 2006). In this study the protocol as shown in Fig. 3 was used.

Among patient whom pulmonary embolism was excluded by clinical assessment plus D-dimer testing, only $0.4 \%$ that pulmonary embolism and none has fatal pulmonary embolism. Among patients whom pulmonary embolism was excluded by $\mathrm{CT}$ angiography, only $0.5 \%$ has fatal pulmonary embolism. In conclusion, this study shows that this algorithm can be used to exclude pulmonary embolism. Clinician needs to be aware that D-dimer assay used in this study is ELISA assay.

\section{CONCLUSION}

Pulmonary embolism is a life threatening condition. Early diagnosis of this condition can greatly improve survival and decrease morbidity. The diagnosis of this condition is very difficult due to non specific history and physical finding, the need for clinical algorithm in selecting the diagnostic test which include imaging study and serum chemistry and clear understanding of advantage and disadvantage of each tests. Physicians must judiciously apply the diagnostic algorithm when making a decision to send patient for diagnostic study. Failure to apply this clinical algorithm may lead to miss diagnosis or overutilization of diagnostic study which might pose harm to the patients.

\section{REFERENCES}

Belle, A.V., H.R. Buller, M.V. Huisman, P.M. Huisman and K. Kaasjager et al., 2006.Effectiveness of managing suspected pulmonary embolism using an algorithm combining clinical probability, D-dimer testingand computed tomography. JAMA, 295: 172-179. PMID: 16403929

Bjork, L. and B.J. McNeil, 1977. Blood flow in pulmonary and bronchial arteries in acute experimental pneumonia and pulmonary embolism. Acta Radiol. Diagn. (Stockh), 18: 393-399. PMID: 920230

Chan, T.C., G.M. Vilke, M. Pollack and W.J. Brady, 2001. Electrocardiographic manifestations: Pulmonary embolism. J. Emerg. Med., 21: 263270. PMID: 11604281

Greenspan, R.H., C.E. Ravin, S.M. Polansky and T.C. McLoud, 1982. Accuracy of the chest radiograph in diagnosis of pulmonary embolism. Invest. Radiol., 17: 539-543. PMID: 7152856

Krishnan, A.S. and T. Barrett, 2012. Westermark sign in pulmonary embolism. N. Engl. J. Med., 366: e16-e16.

Miniati, M., M. Pistolesi, C. Marini, G. Di Ricco and B. Formichi et al., 1996. Value of perfusion lung scan in the diagnosis of pulmonary embolism: Results of the Prospective Investigative Study of Acute Pulmonary Embolism Diagnosis (PISA-PED). Am. J. Respir. Crit. Care Med., 154: 1387-1393. PMID: 8912753 
PIOPED, 1990. Value of the ventilation/perfusion scan in acute pulmonary embolism. Results of the prospective investigation of pulmonary embolism diagnosis (PIOPED). The PIOPED Investigators. JAMA, 263: 2753-2759. PMIID: 2332918

Righini, M., A. Perrier, P.D. Moerloose and H. Bounameaux, 2008. D-Dimer for venous thromboembolism diagnosis: 20 years later. J. Thromb. Haemost., 6: 1059-1071. PMID: 18419743

Ryu, J.H., E.J. Olson, P.A. Pellikka, 1998. Clinical recognition of pulmonary embolism: Problem of unrecognized and asymptomatic cases. Mayo. Clin. Proc., 73: 873-879. PMID: 9737225

Schoepf, U.J., S.Z. Goldhaber and P. Costello, 2004. Spiral computed tomography for acute pulmonary embolism. Circulation, 109: 2160-2167. PMID: 15136509

Stein, P.D., M.L. Terrin, C.A. Hales, H.I. Palevsky and H.A. Saltzman et al., 1991. Clinical, laboratory, roentgenographic and electrocardiographic findings in patients with acute pulmonary embolism and no pre-existing cardiac or pulmonary disease. Chest., 100: 598-603. PMID: 1909617
Stein, P.D., R.D. Hull, K.C. Patel andR.E. Olson,2004. D-Dimer for the exclusion of acute venous thrombosis and pulmonary embolism: A systematic review. Ann. Intern. Med., 140: 589-602.

Stein, P.D., S.E. Fowler, L.R. Goodman, A. Gottschalk and C.A. Hales et al., 2006. Multidetector computed tomography for acute pulmonary embolism. N. Engl. J. Med., 354: 2317-2327. PMID: 16738268

Turkstra, F., P.M. Kuijer, E.J. Van Beek, D.P. Brandjes and J.W.T. Cate et al., 1997. Diagnostic utility of ultrasonography of leg veins in patients suspected of having pulmonary embolism. Ann. Intern. Med., 126: 775-781. PMID: 9148650

Vanni, S., G. Polidori, R. Vergara, G. Pepe and P. Nazerian et al., 2009. Prognostic value of ECG among patients with acute pulmonary embolism and normal blood pressure. Am. J. Med., 122: 257264. PMID: 19272487

Worsley, D.F., A. Alavi, J.M. Aronchick, J.T. Chen andR.H. Greenspan et al., 1993. Chest radiographic findings in patients with acute pulmonary embolism: Observations from the PIOPED Study. Radiology, 189: 133-136. PMID: 8372182 